\title{
Diabetic Nephropathy in Spontaneously Diabetic Torii (SDT) Rats
}

\author{
Takeshi Ohta* and Tomohiko Sasase
}

\begin{abstract}
Biological/Pharmacological Research Laboratories, Central Pharmaceutical Research Institute, Japan Tobacco Inc., 11 Murasaki-cho, Takatsuki, Osaka 569-1125, Japan
\end{abstract}

\begin{abstract}
Diabetic nephropathy is a serious complication of diabetes mellitus, making it essential to develop animal models of diabetic complications to simulate the pathogenesis in humans. We monitored pathobiochemical parameters and histopathological findings of kidneys in male Spontaneously Diabetic Torii (SDT) rats. Renal-related parameters in SDT rats have deteriorated with the progress of diabetes mellitus, and histopathological changes in the renal tubules and the glomeruli were observed with aging. Glycemic control in SDT rats prevented the development of renal lesions. The features of SDT rat indicate its usefulness as an animal model for investigating diabetic nephropathy.
\end{abstract}

Keywords: Diabetic nephropathy, insulin, SDT rat.

\section{INTRODUCTION}

Diabetic nephropathy is the most common cause of endstage renal failure $[1,2]$. From a clinical perspective, it is characterized by the onset of proteinuria, a subsequent decline in glomerular filtration rate, and ultimate progression to renal failure, which is fatal if left untreated [3, 4]. The identification of an experimental animal model with diabetic nephropathy that closely mirrors human disease will significantly enhance our understanding of the nephropathy and accelerate our progress toward more effective treatments.

\section{ANIMAL MODEL}

The functional and morphological features of the diabetic renal lesions have been evaluated in male Spontaneously Diabetic Torii (SDT) rats from 8 to 68 weeks of age [5]. Sprague-Dawley (SD) rats of similar age were used as control animals.

\section{KIDNEY FUNCTION}

In SDT rats, urinalysis showed increase in urine volume and in urinary protein at 24 weeks of age, and further increase thereafter, until 54 weeks of age. Also, an increase of urinary albumin excretion was observed during the same period. The levels of creatinine clearance were higher from 28 to 52 weeks of age, as compared with SD rats.

\section{KIDNEY SIZE}

Renal hypertrophy and glomerular hypertrophy are seen at the early stage of diabetes mellitus. In streptozotocininduced diabetic models, the glomerular hypertrophy was prominent during the first few days of diabetes [6,7]. Also, in SDT rats, the increase of kidney weight and glomerular size was observed from the early stage of diabetes mellitus. Kidney weights in SDT rats were observed from 12 to 20

*Address correspondence to this author at the Japan Tobacco, Inc. Central Pharmaceutical Research Institute 1-1, Murasaki-cho, Takatsuki, Osaka, 569-1125, Japan; Tel: +81-72-681-9700; Fax: +81-72-681-9722;

E-mail: takeshi.ota@jt.com weeks of age. The relative kidney weights significantly increased after 14 weeks of age, as compared with those in age-matched SD rats (Table 1). Moreover, a strong positive correlation was observed between serum glucose level and absolute or relative kidney weight in SDT rats (glucose vs absolute kidney weight: $\mathrm{R}^{2}=0.8233$, glucose vs relative kidney weight: $\left.\mathrm{R}^{2}=0.8121\right)$. Glomerular size in SDT rats and SD rats was determined at 16 weeks of age. The glomerular size in SDT rats was significantly enlarged, as compared with that in SD rats (SDT rats: $9033.7 \pm 666.4 \mu \mathrm{m}^{2}$ vs SD rats: $7020.9 \pm 463.6 \mu \mathrm{m}^{2}$, Data represent means $\pm \mathrm{SD}(\mathrm{n}=4$ or 5)). In streptozotocin-induced diabetic models, kidney weights increased by $15-20 \%$ during the first 4 to 5 days of diabetes and by 70 to $90 \%$ after 6 weeks [6].

\section{HISTOPATHOLOGY}

Histopathological examination of the kidneys revealed changes in the renal tubules from 24 weeks of age, and in the glomeruli, from 32 weeks of age. In the glomeruli, basement membrane thickening was observed from 32 weeks of age, and the change progressed with aging. Mesangial matrix proliferation was observed from 50 weeks of age. These findings included an increase in periodic acid Schiff (PAS) positive areas, fibrous proliferation on Masson's trichrome (MT) staining, and increased type IV collagen positive areas on immunohistochemical (IHC) staining (Fig. 1). The glomerular changes gradually progressed with aging, with diffuse glomerular lesions at 50 and 68 weeks of age. At 68 weeks of age, nodular lesions were observed in a few glomerular capillary loops on PAS staining (Fig. 2). In the renal tubules, glycogen deposition in the tubular epithelium (Armanni-Ebstein lesions) and tubular dilation were noted starting at 24 weeks of age in SDT rats. There were increased hyaline casts at 50 weeks of age. These renal tubular changes markedly progressed with aging, and severe changes throughout the kidneys were present by 68 weeks of age (Fig. 3). At 50 and 68 weeks of age, tubules with glycogen deposition were seen throughout the renal cortex. 
Table 1. Bodyweights, Serum Parameters, and Organ Weights in Male SD Rats and SDT Rats

\begin{tabular}{|c|c|c|c|c|c|}
\hline & \multicolumn{5}{|c|}{ Age (Weeks of Age) } \\
\hline & 12 & 14 & 16 & 18 & 20 \\
\hline \multicolumn{6}{|c|}{ Body weight (g) } \\
\hline SD rat & $567.0 \pm 31.4$ & $565.4 \pm 32.8$ & $634.4 \pm 62.7$ & $635.6 \pm 43.3$ & $691.0 \pm 35.1$ \\
\hline SDT rat & $515.2 \pm 14.2 *$ & $533.6 \pm 14.1$ & $532.9 \pm 12.2^{*}$ & $542.1 \pm 28.9^{*}$ & $508.1 \pm 39.7 * *$ \\
\hline \multicolumn{6}{|c|}{ Glucose $(\mathrm{mg} / \mathrm{dL})$} \\
\hline SDT rat & $297.8 \pm 204.3$ & $347.4 \pm 196.4$ & $450.8 \pm 252.4$ & $473.3 \pm 252.1$ & $604.0 \pm 261.3^{*}$ \\
\hline \multicolumn{6}{|c|}{ Trigryceride (mg/dL) } \\
\hline SD rat & $371.5 \pm 67.1$ & $275.9 \pm 61.3$ & $341.7 \pm 112.0$ & $345.6 \pm 175.8$ & $419.9 \pm 208.1$ \\
\hline SDT rat & $287.2 \pm 125.5$ & $329.7 \pm 93.4$ & $243.3 \pm 52.0$ & $317.1 \pm 122.5$ & $427.9 \pm 127.7$ \\
\hline \multicolumn{6}{|c|}{ Relative kidney weight (mg/g) } \\
\hline SD rat & $5.52 \pm 0.66$ & $5.27 \pm 0.20$ & $5.07 \pm 0.31$ & $4.60 \pm 0.15$ & $4.61 \pm 0.34$ \\
\hline SDT rat & $6.55 \pm 0.77$ & $6.33 \pm 0.68^{*}$ & $6.94 \pm 1.06^{*}$ & $6.92 \pm 1.19^{*}$ & $8.13 \pm 1.64 * *$ \\
\hline
\end{tabular}

Data represent means $\pm \mathrm{SD}(\mathrm{n}=4$ or 5$)$.

$* \mathrm{P}<0.05, * * \mathrm{P}<0.01 v s$ age-matched $\mathrm{SD}$ rats
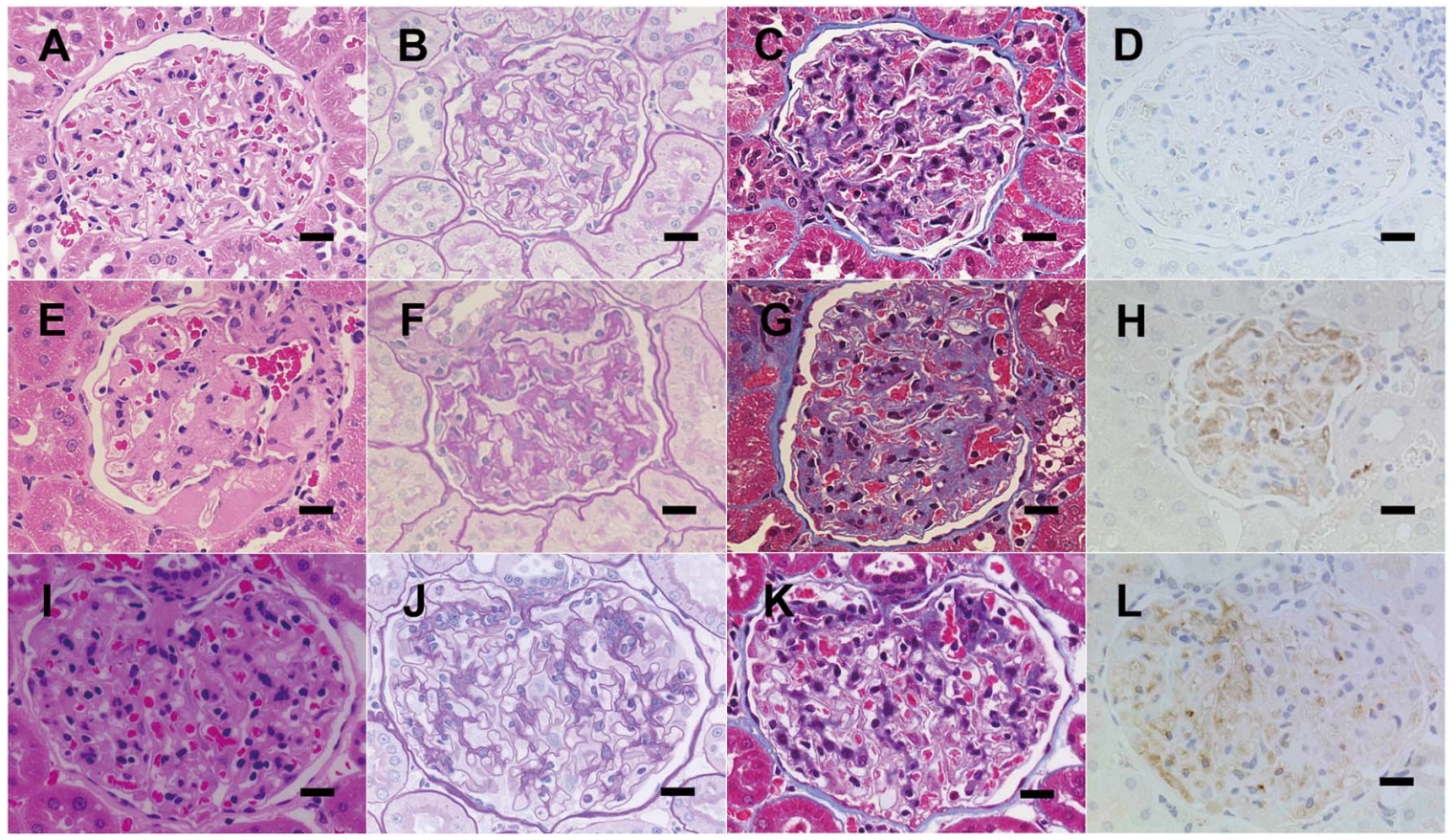

Fig. (1). Histological and immunohistological analysis of glomeruli in SD, SDT, and SDT+insulin rats [5]. A, E, and I: HE stained sections; $\mathbf{B}, \mathbf{F}$, and $\mathbf{J}$ : PAS stain; $\mathbf{C}, \mathbf{G}$, and $\mathbf{K}$ : MT stain; $\mathbf{D}, \mathbf{H}$, and $\mathbf{L}$ : Collagen type IV immunohistostained sections. Kidney sections are from SD (A-D) and SDT rats (E-H) at 50 weeks of age. SDT rats treated with insulin pellets (I-L) at 68 weeks of age. Bar $=20 \mu \mathrm{m}$.

Human clinical stage of diabetic nephropathy is classified from Stage I into Stage V (Stage I: initial stage, II: early renal involvement, III: incipient nephropathy, IV: overt nephropathy, V: end-stage renal disease) [1]. In Stage I, glomerular filtration rate (GFR) is increased, and glomerular hypertrophy and increased kidney volume are observed. In
Stage II, the glomerular basement membrane (GBM) thickening and mesangial expansion are observed. Further GBM thickening and mesangial expansion are shown in Stage III, and albumin excretion rate is increased. Persistent dipstick albuminuria defines overt nephropathy (Stage IV). It is characterized by a decline in GFR and increased mortality. 
The histologic features are diffuse and/or nodular glomerulosclerosis and hypertension is observed. In Stage V, GFR is reduced and glomerular closure and obsolescence are observed. In SDT rat from 24 to 68 weeks of age, an increase of urinary albumin excretion, GBM thickening and mesangial expansion were observed. Those changes are similar to the features as stage I to III in human clinical diabetic nephropathy. SDT rat is considered to be a new model of early phase of diabetic nephropathy.

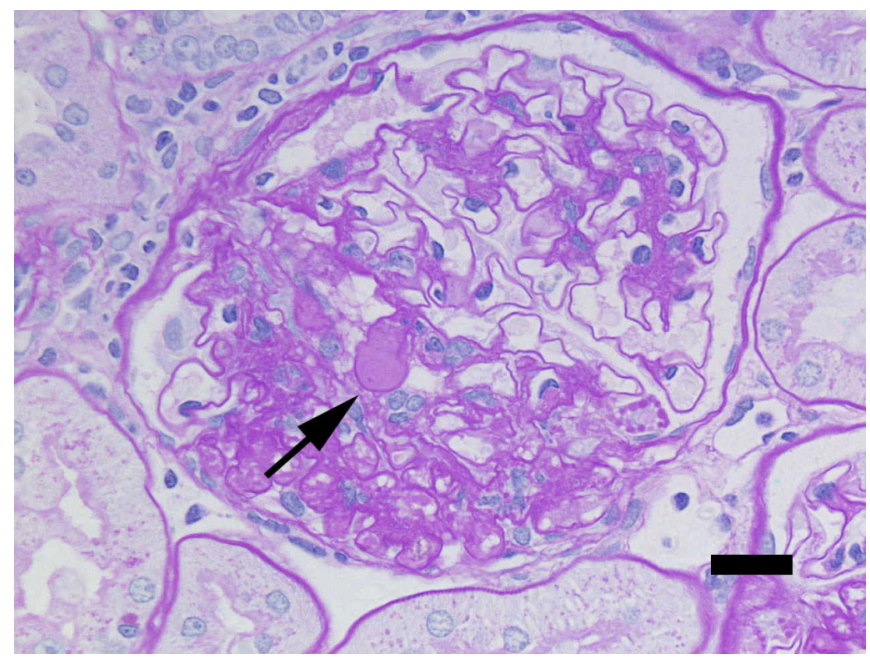

Fig. (2). Light microscopic feature of glomerulus in SDT rat at 68 weeks of age [5]. PAS stain. Bar $=20 \mu \mathrm{m}$. Nodular lesions were observed in a few glomeruli (arrow).

Renal lesions with diabetes mellitus have been reported in other genetic diabetes models. Goto-Kakizaki (GK) rats, a nonobese type 2 diabetes model, exhibited proteinuria by 6 months of age, accompanied by renal histologic abnormalities such as focal glomerulosclerosis, mesangial matrix expansion, and thickening of basement membranes. Furthermore, diffuse global glomerulosclerosis with nodule formation and arteriolar hyalinosis were exhibited by 18 months of age [8,9]. In Zucker Diabetic Fatty (ZDF) rats, an obese type 2 diabetes model, proteinuria started to rise until 3 months of age. The glomerulosclerosis commenced as early as 5 months of age, and was associated with glomerular hypertrophy and mild mesangial expansion [10]. Moreover, an adjacent tubulus showed enlarged cells with clear cytoplasm (Armanni-Ebstein cells), indicating a history of diabetes and glucosuria [11].

\section{EFFECT OF BLOOD GLUCOSE CONTROL}

The main clinical associations that frequently precede overt diabetic nephropathy are poor glycemic control and hypertension [12]. To control blood glucose levels in SDT rats, an insulin pellet was implanted subcutaneously. The rats were then examined to determine whether the renal dysfunction was caused by hyperglycemia [5]. In the insulin treated group, the values for urinary parameters such as urine volume, urine protein, and urinary albumin excretion were clearly decreased (Table 3). Furthermore, the insulin-treated group of SDT rats at 50 and 68 weeks of age showed no glomerular or tubular lesions (Figs. 1 and 3). Renal damage in the SDT rats was obviously prevented by glycemic control.

\section{BLOOD PRESSURE}

There has been no report of examination of the blood pressure in SDT rats. We previously examined the systolic blood pressure (SBP) by the indirect tail cuff method, and observed that the levels at 24 weeks of age tended to increase (SDT rats: $145 \pm 13 \mathrm{mmHg} v s$ SD rats: $125 \pm 21 \mathrm{mmHg}(\mathrm{n}=5)$ ). Table 2 shows the physiological parameters, including blood chemical parameters, renal-related parameters, and blood pressure, in SD rats and SDT rats at that time. Renal-related parameters, such as blood urea nitrogen, urine volume, urine protein and creatinine clearance, were deteriorated with an elevation of blood glucose levels. Moreover, urine angiotensinogen levels in SDT rats at 24 weeks of age tended to elevate (SDT rats: $30.68 \pm 21.93 \mathrm{ng} / \mathrm{mL} v s$ SD rats: $6.84 \pm 2.46 \mathrm{ng} / \mathrm{mL}$ $(\mathrm{n}=5))$. Thus, the increase of blood pressure might be caused by the activation of Renin-Angiotensin System (RAS) [13]. In further study, it is necessary to examine the participation of the blood pressure in diabetic nephropathy of SDT rats.

\section{CONCLUSION}

Renal-related parameters in SDT rats were deteriorated with the progress of diabetes mellitus, and histopathological changes, such as the diffuse glomerular lesions and the nodular lesions, were observed with aging. The histological changes in the kidney of SDT rat mimics the changes seen in the kidney of patients with diabetes. SDT rat is a useful model for investigating diabetic nephropathy. Use of SDT rats will assist in the further elucidation of the pathogenesis of human diabetic nephropathy and in discovering new drugs.
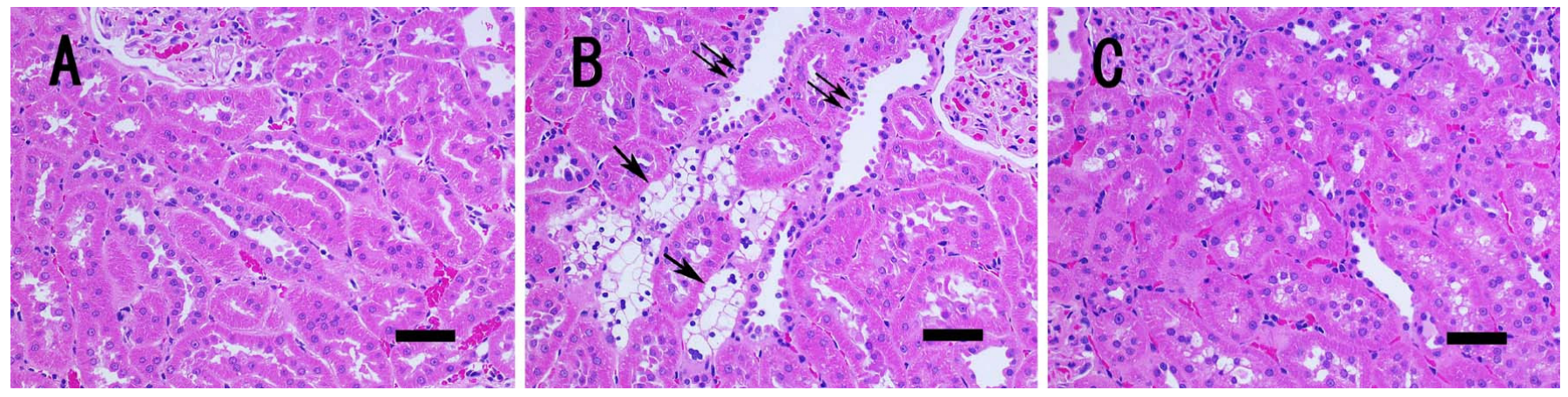

Fig. (3). HE stained section of the kidneys. SD rat (A), SDT rat (B), and SDT rat treated with insulin pellets (C) at 68 weeks of age [5]. Bar = $50 \mu \mathrm{m}$. In the renal tubules of SDT rats, glycogen deposition in the tubular epithelium (single arrow) and tubular dilation (double arrow) were observed. 
Table 2. Physiological Parameters in SD Rats and SDT Rats

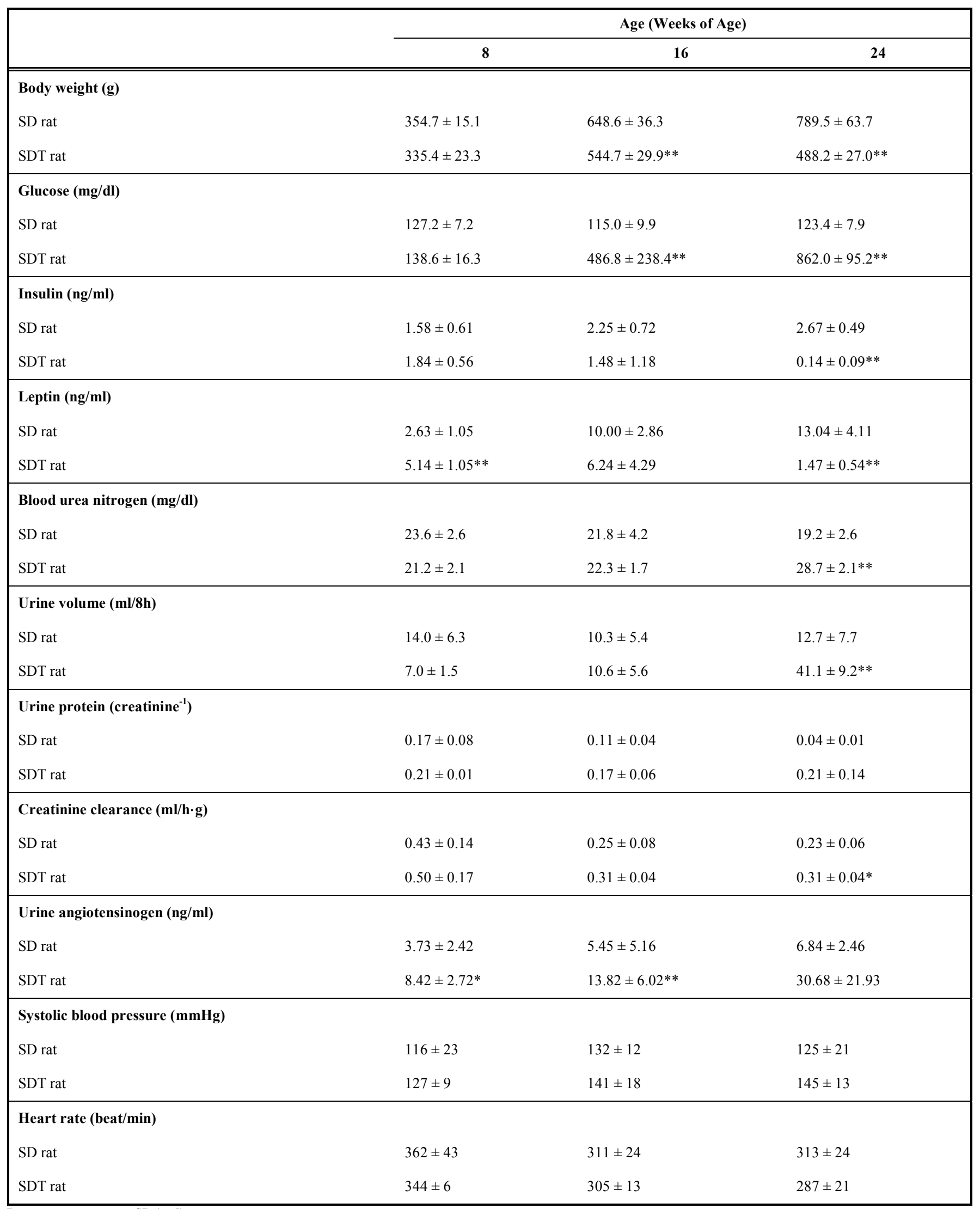


Table 3. Blood Glucose Levels and Urinary Parameters in SD rats, SDT Rats and SDT Rats with Insulin Therapy

\begin{tabular}{|c|c|c|c|}
\hline & \multicolumn{3}{|c|}{ Age (Weeks of Age) } \\
\hline & 36 & 44 & 56 \\
\hline \multicolumn{4}{|l|}{ Glucose (mg/dl) } \\
\hline SD rat & $134.7 \pm 9.2$ & $142.7 \pm 29.0$ & $135.3 \pm 18.7$ \\
\hline SDT rat & $822.1 \pm 134.1 * *$ & $784.3 \pm 118.1 * *$ & $972.3 \pm 149.5 * *$ \\
\hline SDT rat + Insulin & $199.8 \pm 130.3^{\# \#}$ & $189.1 \pm 88.7^{\# \#}$ & $153.0 \pm 18.5^{\ldots \#}$ \\
\hline \multicolumn{4}{|c|}{ Urine volume (ml/24h) } \\
\hline SD rat & $13.7 \pm 2.7$ & $24.2 \pm 9.2$ & $24.0 \pm 8.4$ \\
\hline SDT rat & $253.5 \pm 40.5 * *$ & $307.8 \pm 46.4^{* *}$ & $355.0 \pm 34.2 * *$ \\
\hline SDT rat + Insulin & $28.9 \pm 13.3^{\# \#}$ & $28.3 \pm 10.5^{\# \#}$ & $20.8 \pm 6.5^{\# \#}$ \\
\hline \multicolumn{4}{|c|}{ Urine protein (mg/24h) } \\
\hline SD rat & $29.9 \pm 8.3$ & $46.7 \pm 22.8$ & $56.3 \pm 26.5$ \\
\hline SDT rat & $162.7 \pm 72.2 * *$ & $218.0 \pm 81.1 * *$ & $232.9 \pm 85.8^{*}$ \\
\hline SDT rat + Insulin & $40.0 \pm 14.2^{\# \#}$ & $55.8 \pm 29.0^{\# \#}$ & $54.7 \pm 37.6^{\# \#}$ \\
\hline \multicolumn{4}{|c|}{ Urine albumin excretion (mg/24h) } \\
\hline SD rat & $4.1 \pm 3.6$ & $4.8 \pm 4.5$ & $4.6 \pm 2.3$ \\
\hline SDT rat & $18.2 \pm 12.2^{* *}$ & $14.1 \pm 4.1 * *$ & $16.5 \pm 1.8^{*}$ \\
\hline SDT rat + Insulin & $6.9 \pm 5.1^{\#}$ & $6.9 \pm 4.7^{\# \#}$ & $5.0 \pm 3.6^{\# \#}$ \\
\hline \multicolumn{4}{|c|}{ Creatinine clearance $(\mathrm{ml} / \mathrm{min} / \mathrm{kg})$} \\
\hline SD rat & $1.4 \pm 0.4$ & $4.5 \pm 1.1$ & $4.7 \pm 0.8$ \\
\hline SDT rat & $7.1 \pm 2.5^{* *}$ & $8.1 \pm 1.3 * *$ & $5.9 \pm 2.7$ \\
\hline SDT rat + Insulin & $3.2 \pm 1.9^{\# \#}$ & $3.9 \pm 1.1^{\# \#}$ & $5.2 \pm 1.8$ \\
\hline
\end{tabular}

Insulin therapy was started from 24 weeks of age.

Data represent means $\pm \mathrm{SD}(\mathrm{n}=4-10)$.

${ }^{*} \mathrm{P}<0.05,{ }^{*} \mathrm{P}<0.01$ vs age-matched $\mathrm{SD}$ rats. ${ }^{*} \mathrm{P}<0.05,{ }^{*} \mathrm{P}<0.01$ vs $\mathrm{SDT}$ rats.

\section{REFERENCES}

[1] Ismail N, Becker B, Strzelczyk P, Ritz E. Renal disease and hypertension in non-insulin-dependent diabetes mellitus. Kidney Int 1999; 55: 1-28.

[2] Suzuki Y, Arakawa M. The treatment of the uraemic diabetic. Are we doing enough? A view from Japan. Fumitake Gejyo Collaborate Study Group. Nephrol Dial Transplant 1995; 10(Suppl 7): 47-55.

[3] Calcutt NA, Cooper ME, Kern TS, Schmidt AM. Therapies for hyperglycaemia-induced diabetic complications: from animal models to clinical trials. Nat Rev Drug Discov 2009; 8: 417-29.

[4] Hostetter TH. Diabetic nephropathy. Metabolic versus hemodynamic considerations. Diabetes Care 1992; 15: 1205-15.

[5] Ohta T, Matsui K, Miyajima K, et al. Effect of insulin therapy on renal changes in spontaneously diabetic Torii rats. Exp Anim 2007; 56: $355-62$.

[6] Seyer-Hansen K. Renal hypertrophy in experimental diabetes mellitus. Kidney Int 1983; 23: 643-6.
[7] Seyer-Hansen K. Renal hypertrophy in experimental diabetes: some functional aspects. J Diabet Complications 1987; 1: 7-10.

[8] Nobrega MA, Fleming S, Roman RJ, et al. Initial characterization of a rat model of diabetic nephropathy. Diabetes 2004; 53: 735-42.

[9] Sato N, Komatsu K, Kurumatani H. Late onset of diabetic nephropathy in spontaneously diabetic GK rats. Am J Nephrol 2003; $23: 334-42$.

[10] Hoshi S, Shu Y, Yoshida F, et al. Podocyte injury promotes progressive nephropathy in zucker diabetic fatty rats. Lab Invest 2002; 82: 25-35.

[11] Schafer S, Schmidts HL, Bleich M, Busch AE, Linz W Nephroprotection in Zucker diabetic fatty rats by vasopeptidase inhibition is partly bradykinin B2 receptor dependent. $\mathrm{Br} \mathrm{J}$ Pharmacol 2004; 143: 27-32.

[12] Breyer MD, Bottinger E, Brosius FC, 3rd, et al. Mouse models of diabetic nephropathy. J Am Soc Nephrol 2005; 16: 27-45.

[13] Rahmouni K, Correia ML, Haynes WG, Mark AL. Obesityassociated hypertension: new insights into mechanisms Hypertension 2005; 45: 9-14.

(C) Ohta and Sasase; Licensee Bentham Open.

This is an open access article licensed under the terms of the Creative Commons Attribution Non-Commercial License (http://creativecommons.org/licenses/by-nc/3.0/) which permits unrestricted, non-commercial use, distribution and reproduction in any medium, provided the work is properly cited. 